\title{
ATMOSPHERE MODELS FOR VERY LOW MASS STARS, BROWN DWARFS AND EXOPLANETS
}

\section{BARAFFE}

Ecole Normale Supérieure, CRAL, 69364 Lyon Cedex 07, France

\author{
AND \\ F. ALLARD \\ Dept. of Physics, Wichita State University, Wichita, KS 67260- \\ 0032
}

\section{Introduction}

Over the past decade considerable effort, both observational and theoretical, has been directed towards a more accurate determination of the stellar lower main sequence and of the sub-stellar domain covered by Brown Dwarfs and Planets. Astronomers have been looking for brown dwarfs for more than a decade, either with standard astronomical technics or with microlensing experiments. A breakthrough in the search for brown dwarfs was very recently achieved with the discovery of the first cool brown dwarf GL 229B (Nakajima et al. 1995). At the same epoch, the search for planets blossomed with the discovery of a Jupiter - mass companion of the star 51 Pegasi (Mayor and Queloz 1995). Now, the number of faint, cool stars and substellar objects is rising rapidly.

The most reliable way to identify the nature of observed objects is a direct comparison of observed and synthetic spectra, to determine the effective temperature and metallicity. The mass requires interior models and is determined from consistent evolutionary models (cf. §2). Until recently, little about the atmospheres and spectral characteristics of such cool objects was understood.

T.R. Bedding et al. (eds.), Fundamental Stellar Properties: The Interaction between Observation and Theory, 227-233. (C) 1997 IAU. Printed in the Netherlands. 


\section{Characteristics of cool dwarf model atmospheres}

Very low mass stars (VLMS) or M-dwarfs are characterized by effective temperatures from $\sim 5000 \mathrm{~K}$ down to $2000 \mathrm{~K}$ and surface gravities $\log g \approx$ $3.5-5.5$, whereas brown dwarfs (BD) and extra-solar giant planets (EGP) can cover a much cooler temperature regime, down to some $100 \mathrm{~K}$. Such low effective temperatures allow the presence of stable molecules $\left(\mathrm{H}_{2}, \mathrm{H}_{2} \mathrm{O}\right.$, $\left.\mathrm{TiO}, \mathrm{VO}, \mathrm{CH}_{4}, \mathrm{NH}_{3}, \ldots\right)$, whose bands constitute the main source of absorption along the characteristic frequency domain. Such particular conditions are responsible for strong non-grey effects and significant departure of the spectral energy distribution from a black body emission. Tremendous progress has been made within the past years to derive accurate non-grey atmosphere models by several groups over a wide range of temperatures and metallicities (Plez et al. 1992; Saumon et al. 1994; Brett 1995 Allard and Hauschildt 1995,1997). A detailed description of the progress in the field is given in Allard et al. (1997).

The current grids of atmosphere models now available do not however include the condensation of molecules into grain, which should affect the atmosphere structure and the spectra of cool objects below $\mathrm{T}_{\text {eff }} \sim 2500$ $\mathrm{K}$ (Tsuji et al. 1996a). A recent breakthrough was achieved by Tsuji et al. (1996a) who first included the grain formation and the grain opacities in non-grey atmosphere models for M-dwarfs and brown dwarfs. New generations of atmosphere models taking into account molecular condensation are now essential for a correct description of substellar objects and are now underway (e.g. Burrows et al. 1996, Allard and Alexander 1997).

Another difficulty inherent to cool dwarf atmopsheres is due to the presence of convection in the optically thin layers. This particularity is due to the molecular hydrogen recombination $\left(\mathrm{H}+\mathrm{H} \rightarrow \mathrm{H}_{2}\right)$ which lowers the adiabatic gradient and favors the onset of convective instability. Since radiative equilibrium is no longer satisfied in such atmospheres, the usual procedure based on $T(\tau)$ relationships to construct grey atmosphere models and to impose an outer boundary condition for the evolutionary models is basically incorrect (cf. Chabrier and Baraffe 1997; Chabrier, this conference). An accurate surface boundary condition based on non-grey atmosphere models is therefore required for evolutionary models.

\section{Theory versus observation}

\subsection{M-DWARFS}

The main improvement performed in the modelling of cool model atmospheres is essentially due to more accurate molecular opacity treatment (e.g opacity sampling method, line by line treatment) and improved molec- 


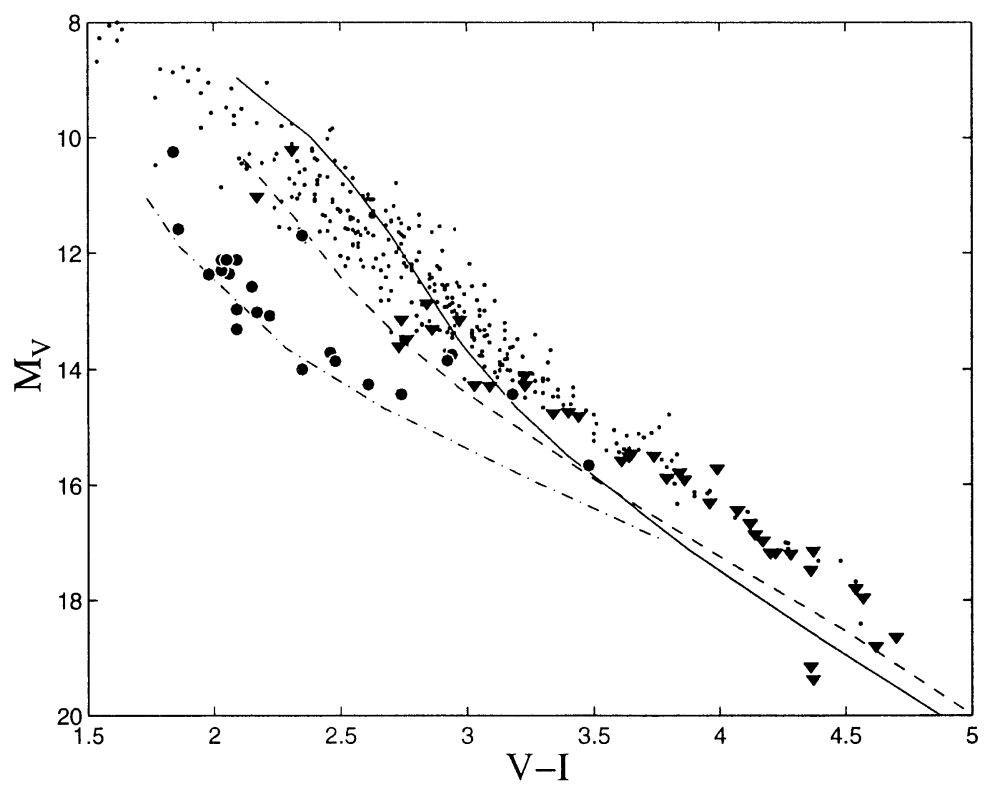

Figure 1. Color - Magnitude diagrams for different metallicity: $[\mathrm{M} / \mathrm{H}]=-1.5$ (dash-dot), $[\mathrm{M} / \mathrm{H}]=-0.5$ (dash) and $[\mathrm{M} / \mathrm{H}]=0$ (solid) from Baraffe et al. (1995). Subdwarf halo field stars from Monet et al. (1992) are indicated by full circles, as well as disk stars of Monet et al. (1992) (full triangles) and Dahn et al. (1995) (dots)

ular data for the main absorbers as $\mathrm{H}_{2} \mathrm{O}$ and $\mathrm{TiO}$ (Jorgensen 1994; Miller et al. 1994; etc...).

The consistent coupling between non-grey atmosphere models and evolutionary models and the use of synthetic spectra to derive the colors provided recently the clue to reproduce the disk and halo field stars in a colormagnitude diagram (cf. Fig. 1). Same agreement is now reached with HST observations of Globular Cluster Main Sequences of low metallicity (Baraffe et al. 1997; see Chabrier 1997, this conference), based on the most recent generation of atmosphere models (Allard et al. 1997). These successful results suggest that the present stellar models are now sufficiently accurate to derive reliable mass-luminosity relationships and thus mass functions, although for solar metallicity a problem remains for the very bottom of the Main Sequence $\left(\leq 0.1 M_{\odot}\right)$.

For solar metallicity, an empirical mass-luminosity relationship has been derived by Henry and McCarthy (1993, HMC93) based on observations of binary systems. Improvement in the molecular linelists of $\mathrm{TiO}$ and $\mathrm{H}_{2} \mathrm{O}$ now leads to a good agreement of the models with the observed relationship in the optical (V-band) and in the near IR (K-band). Figure 2 shows such agreement for stellar models based on recent atmosphere models of Allard 


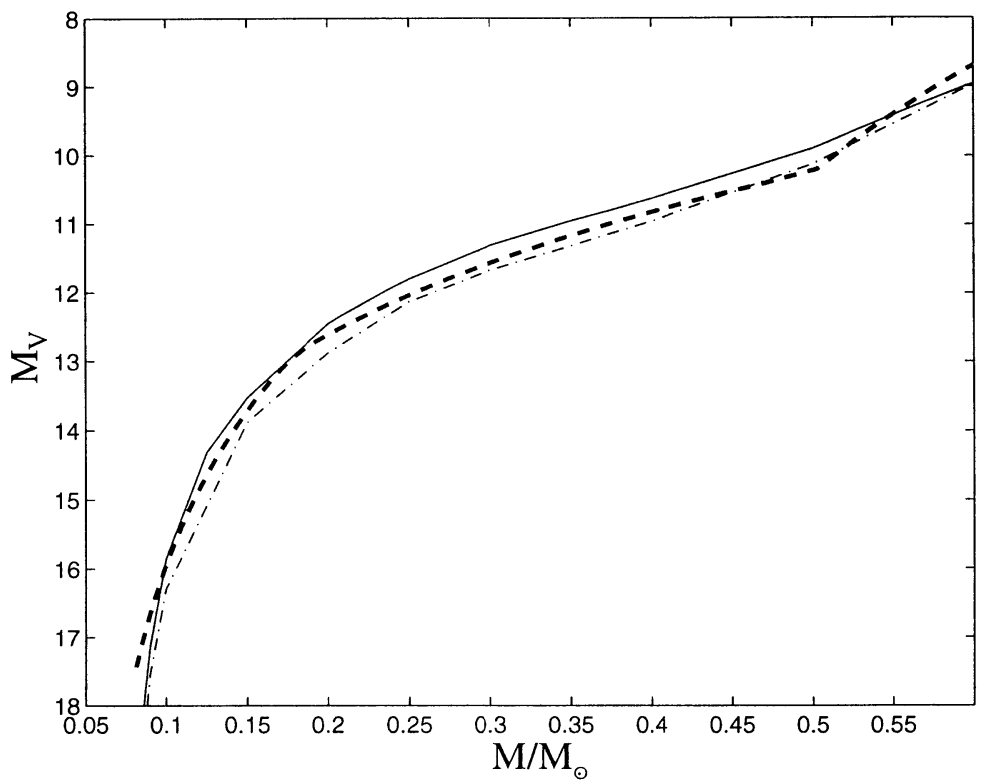

Figure 2. Mass - Magnitude relationship in the V-band. The thick dashed line corresponds to the empirical relationship derived by Henry and McCarthy (1993). Evolutionary models (Chabrier et al. 1996) are based on the Allard and Hauschildt (1997 , solid line) and the Brett-Plez (dash-dot) non-grey atmosphere models.

and Hauschildt (1997) and Brett-Plez (Brett 1995; Chabrier et al. 1996). Note that the change of slope in the HMC93 fit at $\mathrm{M}_{V} \sim 11$ is exagerated and stems from a linear interpolation between remote objects (see Fig. 2 of HMC93).

The comparison of synthetic spectra with observed spectral distribution of M-dwarfs now reaches a good agreement in the IR (cf. Leggett et al. 1996; Allard et al. 1997) for atmosphere models including the new water linelist of Miller et al. (1994). Disagreement still remains in the optical colors (V-I, $\mathrm{R}-\mathrm{I})$ and for the latest-type M-dwarfs $\left(\mathrm{T}_{\text {eff }} \leq 3500 \mathrm{~K}\right)$ where the models systematically overestimate the water band strengh. Such discripancies may be due either to remaining shortcomings in the $\mathrm{TiO}$ (optical) and $\mathrm{H}_{2} \mathrm{O}$ (IR) line lists or to the effect of grain formation, as discussed below.

\subsection{SUBSTELLAR OBJECTS}

As the effective temperature decreases below $\sim 2000 \mathrm{~K}$, the IR spectrum is dominated mainly by $\mathrm{H}_{2} \mathrm{O}, \mathrm{CH}_{4}$ and $\mathrm{NH}_{3}$. The presence of methane is a clear signature of the substellar nature of an object. The most stringent test for extremely cool models was provided recently by the brown dwarf 


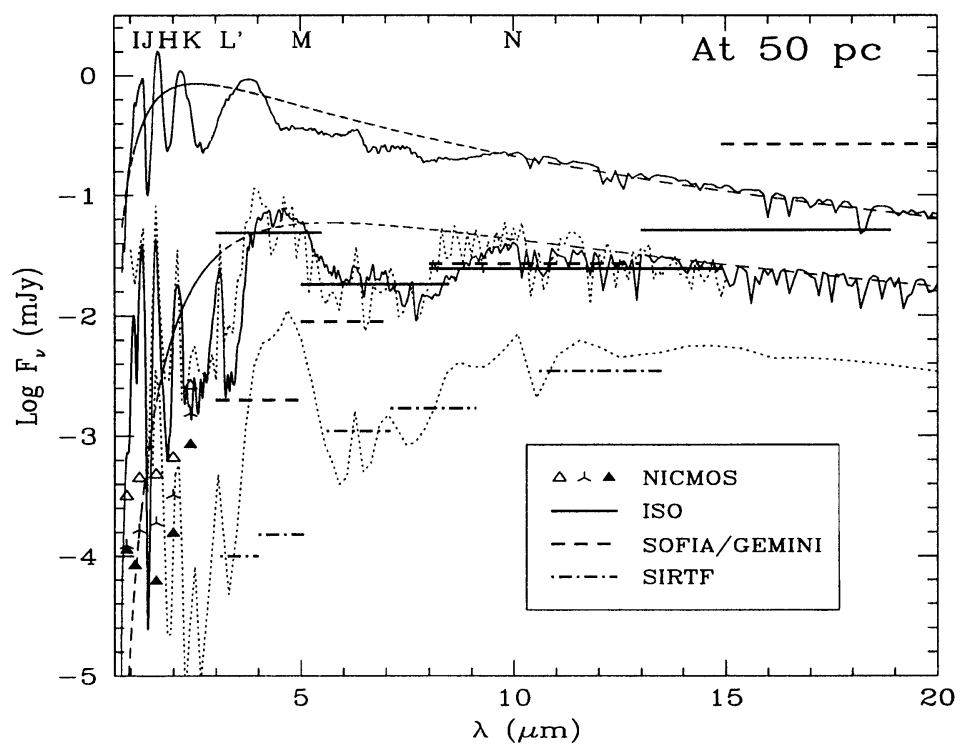

Figure 3. Predicted absolute fluxes of BD or EGP at $50 \mathrm{pc}$ compared to the sensitivity of ground and space-based platforms. Models of both Allard et al. (1996) (solid) and Marley et al. (1996) (dotted) are shown which simulate (i) a brown dwarf near the hydrogen burning limit (topmost spectrum $T_{\text {eff }}=2000 \mathrm{~K}$ ), (ii) an evolved brown dwarf similar to GL 229B (central spectra: $T_{\text {eff }}=900 \mathrm{~K}$ and $960 \mathrm{~K}$ ), and (iii) an extremely cool object (lowermost spectrum: $T_{\text {eff }}=500 \mathrm{~K}$ ). Dashed curves give the corresponding black-body.

Gl 229B (Nakajima et al. 1996). The synthetic spectra calculated by different groups for an effective temperature of $\sim 1000 \mathrm{~K}$ yield to a remarkable agreement with the observed spectrum, reproducing the main molecular absorption features (Allard et al. 1996, Tsuji et al. 1996b, Marley et al. 1996).

The predicted absolute fluxes of BD or EGP (Allard et al. 1997) are illustrated in Fig. 3 and compared to the sensitivity of ground and space-based observing platforms, as given by Saumon et al. (1996). The predictions are shown for different effective temperatures and compared to a black body emission at the same $\mathrm{T}_{\text {eff }}$. Strong departure from the black body emission illustrates the necessity to derive synthetic spectra for this type of objects. As shown in Fig. 3, the flux peaks in the $4.5-5 \mu \mathrm{m}$ window, which seems to be the best region for the search of cool BD or EGP.

Despite the success of modelling GL 229B, the remaining challenge for atmosphere modellers in the low temperature regime is the grain formation. Although the effects of grain absorption appear more subtil in currently available IR spectra of Gl229B (cf. Tsuji et al. 1996b; Allard et al. 


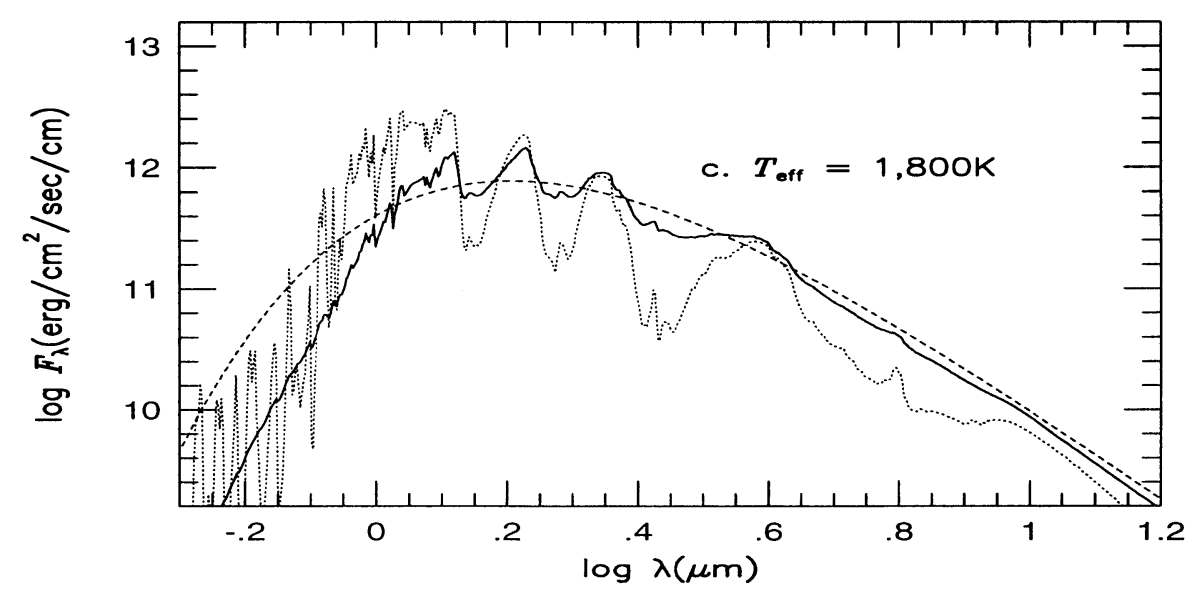

Figure 4. Predicted spectral energy distribution for dusty (solid line) and dust-free (dotted line) models with $\mathrm{T}_{\text {eff }}=1800 \mathrm{~K}$ (from Tsuji et al. 1996a). The black body curve corresponds to the dashed line.

1996, Marley et al. 1996), the inclusion of this effect seems necessary to explain the IR spectra of very late-type M-dwarfs. As shown by Tsuji et al. (1996a) and illustrated in Figure 4, kindly provided by T. Tsuji, dust opacities tend to reduce the molecular absorption feature and could explain the overestimation of water absorption found in dust-free model atmospheres.

\section{Conclusion}

The last few years have shown impressive improvement in the modelization of cool model atmospheres. The main efforts in both theoretical and observational directions lead to a much better understanding of the bottom of the Main Sequence and the substellar regime. Remarkable agreements between theory and observations are now reached on different fronts: color - magnitude diagram, mass - luminosity relationships, spectrum. The success of the theory is most encouraging and shows that it has finally come to maturity. 


\section{References}

Allard, F., and Hauschildt, P. H. (1995): ApJ, 445, 433

Allard, F., Hauschildt, P.H., Baraffe, I., Chabrier, G. (1996): ApJl, 465, L123

Allard, F., and Hauschildt, P. H. (1997): in preparation

Allard, F., and Alexander D. R. (1997): in preparation

Allard, F., Hauschildt, P.H., Alexander D. R., Starrfield, S. (1997): Ann. Rev. A\&A, 35, 137

Baraffe, I., Chabrier, G., Allard, F., Hauschildt P. (1995): ApJl, 446, L35

Baraffe, I., Chabrier, G., Allard, F., Hauschildt P. (1997): A\&A, in press

Brett, J.M. (1995): A\&A, 295, 736

Burrows. A. et al. (1996): Nucl. Phys. B, 51B, 76

Chabrier, G., Baraffe, I., Plez, B. (1996): ApJ, 459, L91

Chabrier, G., Baraffe, I. (1997): A\&A, in press

Dahn, C.C., Liebert, J., Harris, H.C., Guetter, H.H. (1995): The bottom of the mainsequence and below, Ed. C. Tinney

Jorgensen, U. G.. (1994): ApJ, 284, 179

Henry , T.D., and McCarthy, D.W.Jr. (1993): AJ, 106, 773

Leggett. S.K., et al. (1996): ApJ, 104, 117

Marley et al. (1996): Science, 272, 1996

Mayor, M., Queloz, D. (1996): Nature, 378, 355

Miller, S., Tennyson, J., Jones, H.R.A, Longmore, A.J. (1994): in Molecules in the Stellar Environment, ed. U.G Jorgensen, Lecture Notes in Physics.

Monet D.G. et al. (1992): Astron. J., 103, 638

Nakajima et al. (1995): Nature, 378, 463

Plez, B., Brett, J.M., Nordlund, A. (1992): A\&A, 256, 551

Saumon, D., Bergeron, P., Lunine, L.I., Hubbard, W.B., Burrows, A. (1994): ApJ, 424, 333

Saumon, D. et al. (1996): ApJ. 460, 993

Tsuji, T., Ohnaka, K., Aoki, W. (1996a): A\&A, 305, L1

Tsuji, T., Ohnaka, K., Aoki, W., Nakajima. T. (1996b): A\&A, 308, L29

\section{DISCUSSION}

BENGT GUSTAFSSON: Isn't the dilemma with ascribing the apparent saturation of water vapour bands in late $M$ dwarfs that if you ascribe it to dust you have to explain why there are no similar problems for the brown dwarf GI 229B. Or, is there no visible dust there? If so, why not?

ISABELLE BARAFFE: The spectrum of Gl 229B shows no evidence for the presence of dust. Tsuji has shown that dusty models cannot match the observed spectra. Therefore it seems that grains have settled down below the photosphere and their presence cannot be detected. Another possibility is that clouds formed. The question is open.

ANDRE MAEDER: About the inclusion of dust in the atmosphere models, do you mean including dust in the equation of state, in thermodynamics and opacities?

ISABELLE BARAFFE: Yes. 